\title{
ADDITIVE MANUFACTURING OF HETEROGENEOUS LATTICE STRUCTURES: AN EXPERIMENTAL EXPLORATION
}

\author{
Leonardi, Francesco (1); Graziosi, Serena (1); Casati, Riccardo (1); Tamburrino, Francesco (2); \\ Bordegoni, Monica (1)
}

1: Politecnico di Milano, Italy; 2: University of Pisa, Italy

\begin{abstract}
3D printed heterogeneous lattice structures are beam-and-node based structures characterised by a variable geometry. This variability is obtained starting from a periodic structure and modifying the relative density of the unit cells or by combining unit cells having different shapes. While several consolidated design approaches are described to implement the first approach, there are still computational issues to be addressed to combine different cells properly. In this paper, we describe a preliminary experimental study focused on exploring the design issues to be addressed as well as the advantages that this second type of heterogeneous structures could provide. The Three-Point-Bending test was used to compare the behaviour of different types of heterogeneous structures printed using the Fused Deposition Modeling (FDM) technology. Results demonstrated that the possibility of combining multiple unit cells represents a valid strategy for performing a more effective tuning of the material distribution within the design space. However, further studies are necessary to explore the behaviour of these structures and develop guidelines for helping designers in exploiting their potential.
\end{abstract}

Keywords: Design for Additive Manufacturing (DfAM), 3D printing, Lightweight design, Heterogeneous lattice structures

Contact:

Graziosi, Serena

Politecnico di Milano

Department of Mechanical Engineering

Italy

serena.graziosi@polimi.it

Cite this article: Leonardi, F., Graziosi, S., Casati, R., Tamburrino, F., Bordegoni, M. (2019) 'Additive Manufacturing of Heterogeneous Lattice Structures: An Experimental Exploration', in Proceedings of the 22nd International Conference on Engineering Design (ICED19), Delft, The Netherlands, 5-8 August 2019. DOI:10.1017/dsi.2019.71 


\section{INTRODUCTION}

The interest for Additive Manufacturing (AM) technologies is nowadays continuously growing in a wide range of industrial contexts. This growth is a consequence of the advantages provided by these technologies to the product development process (Thompson et al., 2016): increased design freedom; active support to prototyping activities; product customisation even in the final stages of the process without the risk of compromising the manufacturing chain; a shortened manufacturing line and the possibility to reduce assembly steps. The design freedom is reflected in the possibility to generate complex, multi-functional and optimised geometries. That fact is having, inevitably, a profound implication in the way designers work and in the development of the next generation of Design for Additive Manufacturing (DfAM) tools and methods (Rosen et al., 2015; Hague et al., 2003).

A significant amount of research efforts concerning design freedom is concentrated on defining strategies for the proper local tuning of the material distribution on a mesoscale level inside the design space. Such tuning is the local modification of the geometry which directly affects the properties. This is a design possibility which only lattice structures can provide. Indeed, the design and fabrication of lattice/cellular structures (lattice structures are mainly built using beams and nodes) is nowadays a topic of great interest in multiple research communities. As discussed in (Tang and Zhao, 2018), these structures can be: homogeneous and heterogeneous. In homogeneous structures a unique unit cell is periodically patterned within the design space. Heterogeneous structures can be of two types (Tang and Zhao, 2018): the heterogeneous properties are obtained through a graded distribution of the material, but the wireframe of the structure is homogeneous (i.e., a unique type of unit cell is used) or through different unit cell geometries arranged within the same design space. They are both valid strategies for creating a functional gradient inside the structure (Tang and Zhao, 2018).

Computational approaches for designing homogeneous and heterogeneous structures are already available in the literature (e.g., see (Tang and Zhao, 2018) for a detailed analysis). An already explored strategy, is first to perform a topology optimisation (TO) of the design space, to get an initial idea of the optimal material distribution inside it, and then replace such material with a structure. According to Panesar et al. (2018) this structure could be: 1) an intersected lattice, obtained by combining the TO solid solution with a homogeneous lattice structure; 2) a graded lattice (i.e. heterogeneous) whose gradient of density reflects the outputs provided by the TO, but where the values of the Volume Fraction (VF, i.e., the ratio between the volume of the initial design space and the volume of the optimised design space) below a minimum are considered as empty zones, while the VF values above a maximum threshold are considered as fully solid zones; and 3) a scaled lattice that is similar to a graded one, but where the TO outputs are scaled between a minimum and a maximum VF value. The study of Panesar et al. (2018) demonstrated that lattice structures generated from the TO have superior properties compared to uniform lattice structures and that the graded shape is the most promising. The potentialities of graded cellular structures are for example also demonstrated in (Li et al., 2019).

These types of computational approaches, based on optimisation methods, can be automatised, continuously improved and also extended to multi-material structures (e.g., see (Wang, Zhang, Daynes, Zhang, Feih and Wang, 2018; Primo et al., 2017; Stankovic et al., 2015)). As clarified in (Liu et al., 2008; Tang and Zhao, 2018) some methods implement concurrent optimisation strategies: the optimal mesostructure (i.e. the lattice/cellular structure) for the selected design space also represents the optimal material distribution at the macro-scale. However, as already underlined in (Tang and Zhao, 2018), especially in the case of heterogeneous structures made up of different types of unit cells, the connectivity among unit cells is an aspect that still needs further research efforts. Besides, due to the high computational resources required for generating this type of heterogeneous structures, unit cells having similar topologies are considered, while it would be interesting to explore a design method able to exploit the properties of different topologies (Tang and Zhao, 2018). Hence, in light of these considerations, this paper describes the preliminary studies performed to derive a design strategy to be used during the concept design phase of heterogeneous structures, in particular of those characterised by different cell geometries. The objective is to guide designers in performing an initial screening of the unit cells arrangement and thus of the material distribution in order to properly modify the stress distribution inside the structure. ThreePoint Bending (TPB) tests were performed on 3D printed PLA samples of the following types of lattice structures (they are all beam-and-node based): homogeneous (HO), heterogeneous-density (H-D, i.e., 
structures having a unique type of cell, but whose relative density is variable), heterogeneous-cells (H$\mathrm{C}$, i.e., structures with different unit cell geometries) and heterogeneous-cells + density $(\mathrm{H}-\mathrm{C}+\mathrm{D}$, i.e., structures having different unit cell geometries and a variable relative density). These tests were used to compare their behaviours and derive some initial considerations.

The paper is structured as follows: Section 2 explores the concept of heterogeneous lattice structures; Section 3 describes how the lattice samples were designed and tested; Section 4 summarises the main results of the testing activity while Section 5 draws some conclusions.

\section{BACKGROUND}

The idea of creating heterogeneous structures made up of different types of unit cells has already attracted lots of attention in the literature. For example, Watts and Hague (2006) proposed a methodology, tested on cantilever beams, where the TO is combined with Genetic Algorithms (GA) to design structures characterised by a uniform stress distribution, which is what occurs in natural systems. To reach this target, it is fundamental to have a variable material density that can be obtained in two ways: by combining multiple materials or, in case a single material is used, by using unit cells having different relative density values. The automatic combination of multiple unit cells using a GA, as discussed in (Watts and Hague, 2006), is computationally intensive. Indeed, in this work, a unique "layer" of unit cells is considered to fill the design space. Besides, the selection of the unit cells among a pre-defined list, as well as their distribution within the design space, is performed mainly considering the unit cell relative density value as the design variable.

Xiao et al. (2018) also mention the possibility of combining multiple unit cells according to the loading pattern acting on different areas of the structure. However, the approach used in this work is focused on optimising the material distribution of the single voxel and not of the whole structure due to, again, the high computational resources needed for the optimisation.

Another interesting approach focused, as in (Watts and Hague, 2006), on making the stress distribution of the lattice structure as more uniform as possible, is described in (Chougrani et al., 2018). A finite element analysis is performed to guide the suppression of beams that less contribute to the desired mechanical behaviour of the structure. The output of such an approach is an optimised non-uniform patterned lattice structure, as defined by the authors (Chougrani et al., 2018). This type of structure is generated using a single unit cell whose pattern is modified to follow the stress field. The output structure is still not conceived as the combination of different unit cells, but the possibility to suppress beams is, however, a further and relevant design strategy to create heterogeneous lattice structures. This strategy could be complementary to others.

An interesting concurrent topology optimisation strategy able to support the design of heterogeneous multi-material structures is described in (Deng and Chen, 2017). Actually, in this work, the objective is to fill the design space with different porous materials. These materials are obtained through different types of microstructures which are designed by the algorithm and arranged into different regions. Constraints are implemented for taking into account connectivity issues among different microstructures, but mainly for the 2D design space. The connectivity issue still needs further explorations as also underlined in (Tang and Zhao, 2018). Besides, especially for the 3D design space, the geometry of the microstructure and of its unit cells is not clearly defined, due to the complexity of performing this type of computational calculation. An attempt to embed connectivity constraints when generating heterogeneous structures, using concurrent topology optimisation strategies, is discussed by Du et al. (2018). However, in this case, firstly the different regions of the design space are defined and then 2D design spaces are tested. Another interesting concurrent optimisation strategy is discussed in (Wang, Zhu, Zhang, Li and Kong, 2018). The authors describe a parametric approach for creating the optimal microstructure distribution within 2D design spaces. As underlined by the authors these microstructures are not uniform but their unit cells are similar: the different microstructures are all characterised by a square frame. That solution avoids connectivity issues. Finally, the work of Tang and Zhao (2018) extends the potentiality of concurrent topology optimisation strategies by developing an algorithm for generating heterogeneous multi-functional structures (both thermal and structural targets are considered) since previous studies were focused only on load bearing, as underlined in (Tang and Zhao, 2018). However, in this work the heterogeneous structure is obtained by varying the relative density of the structure and using a single unit cell. 
Other relevant examples of heterogeneous structures made up of different types of unit cells are available in the field of metamaterials: as demonstrated in (Kolken et al., 2018), the properties of metamaterials also depend on the complexity of their geometry. Indeed, in (Kolken et al., 2018), the authors describe the development of a new hybrid meta-biomaterial for metal-implants. The new metamaterial is obtained by combining two mechanical meta-biomaterials having opposite Poisson's coefficients. The output of this study is an innovative hybrid metal-implant with improved implant-bone contact. The design space (i.e., the implant) is split into two sub-volumes (i.e., two sub-regions) each one filled with a different type of unit cell. An experimental approach was used to demonstrate the effectiveness of the developed structure.

This brief overview outlines two main aspects. Computational strategies are powerful when designing homogeneous structures and heterogeneous structures having a graded distribution of the material and a single unit cell. In the case of heterogeneous structures characterised by multiple unit cells, there are still several complex and challenging computational issues that need to be overcome such as the definition in detail of the lattice geometry and the possibility to consider connectivity constraints. Besides, in all these approaches the design space is discretised into macro-regions. Hence it is still not possible to consider the single voxel (i.e. the single unit cell). Besides, multiple aspects concur in determining the final behaviour of the structure (Tamburrino et al., 2018) among which the type and the dimensions of the unit cell and the population strategy used (i.e., periodic or conformal). Hence, considering all these open issues, this paper discusses the preliminary steps of a more in-depth experimental study focused on exploring the behaviour of heterogeneous structures. The study aims to derive indications for guiding designers in making more informed decisions when performing the preliminary design of these structures. Parameters such as the mass fraction (MF, i.e., the mass of the lattice structure vs the mass of the solid design space), the beam cross-section dimension and the unit cell type, are explored to compare the behaviour of the different type of heterogeneous lattice structures analysed.

\section{MATERIALS AND METHODS}

This Section describes how the lattice structures were designed and tested. The design space is a beam having the following dimensions: $80 \mathrm{~mm} \times 20 \mathrm{~mm} \times 20 \mathrm{~mm}$ (Figure 1). We selected the TPB test to have different regions of the design space undergoing different types of stress. All the samples were printed using the Ultimaker White PLA (Ultimaker PLA technical data sheet, 2018) filament and the Ultimaker 3 (https://ultimaker.com) FDM printer, with a $0.4 \mathrm{~mm}$ nozzle diameter while the Ultimaker Cura (2019) software 3.6.0 was used to generate the printing instructions. The default printing profiles, defined for this type of material, were used.

\subsection{Lattice structures design}

To design the lattice structures we used the nTopology Element Pro software (www.ntopology.com). The creation of a lattice structure involves two macro steps: "generate" and "thicken". In the first step, the designer has to decide how to discretise the design space, which means selecting the unit cell type, the shape and the X-Y-Z dimensions of the voxel used to perform the discretisation. The output of this discretisation is a wireframe representation (i.e., the skeleton) of the lattice structure. To thicken the structure, there are two possibilities, i.e. performing a uniform or a heterogeneous material distribution. The uniform distribution is obtained by setting the beam thickness, and the output is a homogeneous lattice structure. The graded one can be obtained into two ways: using a geometric modifier, or performing a size optimisation of the beam thickness. When performing this modelling activity the designer has to make several choices, each one influencing the behaviour of the final structure. In this preliminary study, to limit the number of experimental variables, we fixed the dimensions of the voxel (see Table 1): it is a cube, with an edge of $5 \mathrm{~mm}$. This choice allowed us to get an adequate number of unit cells (4 unit cells along $\mathrm{Y}$ and $\mathrm{Z}$, and 16 along $\mathrm{X}$, as shown in Figure 1) and to generate self-supporting structures. Concerning the material distribution, we generated HO, H-D, H-C, H-C+D (see Section 1) lattice structures as summarised in Table 1. The dimensions of the diameter of the beam were selected considering both technological and design requirements. Having an FDM machine with a $0.4 \mathrm{~mm}$ extruder diameter, the minimum value of the beam diameter should not be lower than $0.8 \mathrm{~mm}$. As the maximum beam dimension, we considered $2.2 \mathrm{~mm}$. Finally, the beam diameters of the structures were expressly selected to create structures having a similar MF (see Table 1). It is evident that only the MF of homogeneous 


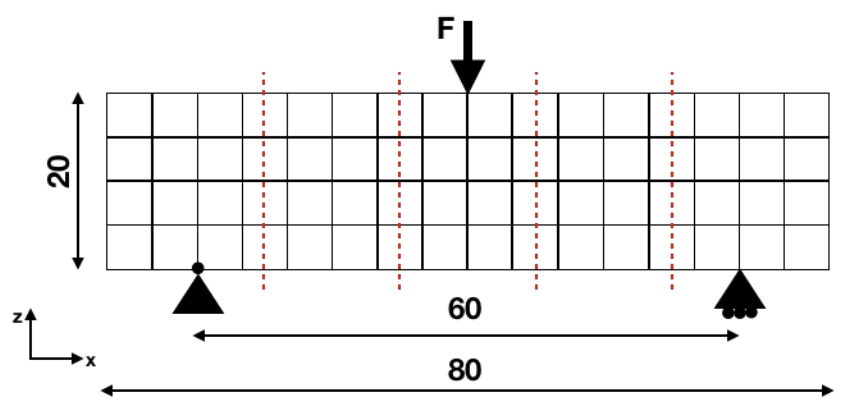

Figure 1. The discretisation of the design space and the boundary conditions defined to simulate the TPB test. The vertical dotted lines show the 5 regions that will be used for creating the $\mathrm{H}-\mathrm{C}$ and $\mathrm{H}-\mathrm{C}+\mathrm{D}$ structures.

structures corresponds to the relative density of the unit cell. That is the reason why working with heterogeneous structures we preferred to consider the MF values.

The unit cells selected for creating the lattice structures are the Face Centered Cubic (FCC) and the Cubic Fluorite (CF). The FCC has 12 beams and 6 nodes (see Figure 2), while the Cubic Fluorite (CF) has 32 beams and 22 nodes (see Figure 2). The FCC cell is isotropic (Xiao et al., 2018) and stretchdominated according to Maxwell's stability criterion (Ashby, 2006); the CF is bending-dominated and isotropic as well. Hence, they are both isotropic but they have opposite behaviour, and they are also characterised by a significantly different number of beams and nodes (12 vs 32 and 6 vs 22 , respectively) which means having a significantly different relative density for the unit cell if the same beam diameter is used. Another important aspect is that they have good connectivity (see Section 2): when translating these two unit cells of half of the voxel length, they can be linked through 4 nodes without generating unwanted free beams (see Figure 2). It is also worth underlying that using these two cells we can also choose the degree of connectivity. Indeed to connect the FCC to the CF, we could use 1 or 4 nodes. We selected the configuration with 4 nodes to have a gradual shift between these two unit cells. However, this is also another design aspect to be investigated. Indeed, the degree of connectivity between two cells determines how the load will be transferred between them. This aspect is not deeply investigated in the literature.

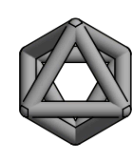

FCC

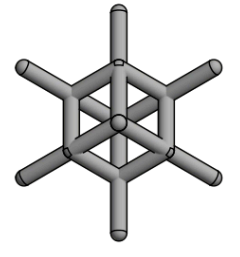

CF

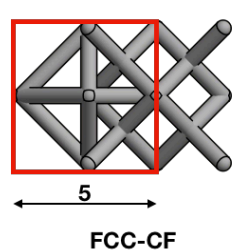

FCC-CF

Figure 2. The unit cells (FCC and CF) used for creating the lattice structures. The unit cell called FCC-CF is the one created at the interface between two different regions to guarantee the connectivity between them (see also Figure 3). The square represents the frontal view of the voxel (5 $\mathrm{mm}$ edge).

For designing the H-C and H-C+D structures, i.e., the MIX, the MIX-Inverted (MIX-I) and the MIXOptimised (MIX-O) samples (Table 1) we used the following approach. The design space was divided into 5 regions (see Figures 1 and 3) filled alternately with the two unit cells (i.e., FCC and CF). The MIXI structure (see Figures 3 and 4a) has the same unit cells but placed inverted with respect to the MIX (see Figure 3). This division into multiple regions (as in (Du et al., 2018)) was, in this case, performed considering the position of loads and constraints (see Figure 1). Besides, the following considerations were also made. Being a preliminary study we decided to compare structures having the same type of behaviour: these regions (see Figure 1) allowed us to guarantee that both homogeneous and heterogeneous structures have symmetric behaviour in term of compression-tension since they all undergo the same bending moment distribution. In a further study, this symmetric behaviour could be modified on purpose, considering a different discretisation. These regions could be seen as functional volumes to which a microstructure is assigned to address specific functional targets. The idea of creating functional 
volumes was also presented in (Tang and Zhao, 2015), but in that study they were used to optimise lattice structure orientations. The more this heterogenisation is performed at the voxel level, the more the behaviour of the structure can be controlled locally. Hence, such discretisation could be theoretically performed at a row and/or column level or even considering the single voxel. Besides, the discretisation of the design space was also performed to guarantee that the three central regions, which are affected by the shift from one type of unit cell to the other, have an integer number of voxels. Another design aspect that was considered, in order to set some correlations among the structures, is that the MF value of the MIX sample (0.25, Table 1$)$ is equal to the average MF of the two homogeneous structures $(0.19$ for FCC_1 and 0.31 for the CF, see Table 1). The MIX sample has the following volume subdivision: $62.5 \%$ of the design space is filled with FCC cells while $37.5 \%$ with CF cells; for the MIX-I (Figure 4a) we got opposite values (i.e., $62.5 \%$ with $\mathrm{CF}$ and $37.5 \%$ with FCC). Some structures were also printed intentionally using the same theoretical resistant cross-section (see Table 1).

The MIX-O (see Table 1 and Figure 4b) and FCC-O samples (see Table 1 and Figure 5) were generated through a size optimisation: the structures were constrained and loaded replicating the TPB testing conditions (see also Figure 1) and using the same diameter variation gap (0.8 - $2.2 \mathrm{~mm})$.

Table 1. Main characteristics of the lattice structures printed to perform the TPB tests (the weight and the MF are mean values obtained printing 3 replica for each type of sample).

\begin{tabular}{lccccr}
\hline Sample & $\begin{array}{c}\text { Beam } \\
\varnothing(\mathrm{mm})\end{array}$ & $\begin{array}{c}\text { Material } \\
\text { Distribution }\end{array}$ & $\begin{array}{c}\text { Voxel } \\
\text { Edge }(\mathrm{mm})\end{array}$ & $\begin{array}{c}\text { Weight } \\
(\mathrm{g})\end{array}$ & MF \\
\hline FCC_1 & 1 & HO & 5 & 7.37 & 0.19 \\
FCC_2 & 1.1 & HO & 5 & 9.40 & 0.24 \\
FCC_3 & 1.3 & HO & 5 & 12.50 & 0.32 \\
CF & 1 & HO & 5 & 12.20 & 0.31 \\
MIX (62.5\%FCC-37.5\%CF) & 1 & H-C & 5 & 9.78 & 0.25 \\
MIX-I (62.5\%CF-37.5\%FCC) & 1 & H-C & 5 & 10.39 & 0.27 \\
MIX-O (62.5\%FCC-37.5\%CF) & $0.8-2.2$ & H-C+D & 5 & 14.25 & 0.37 \\
FCC-O & $0.8-2.2$ & H-D & 5 & 11.73 & 0.30 \\
\hline
\end{tabular}

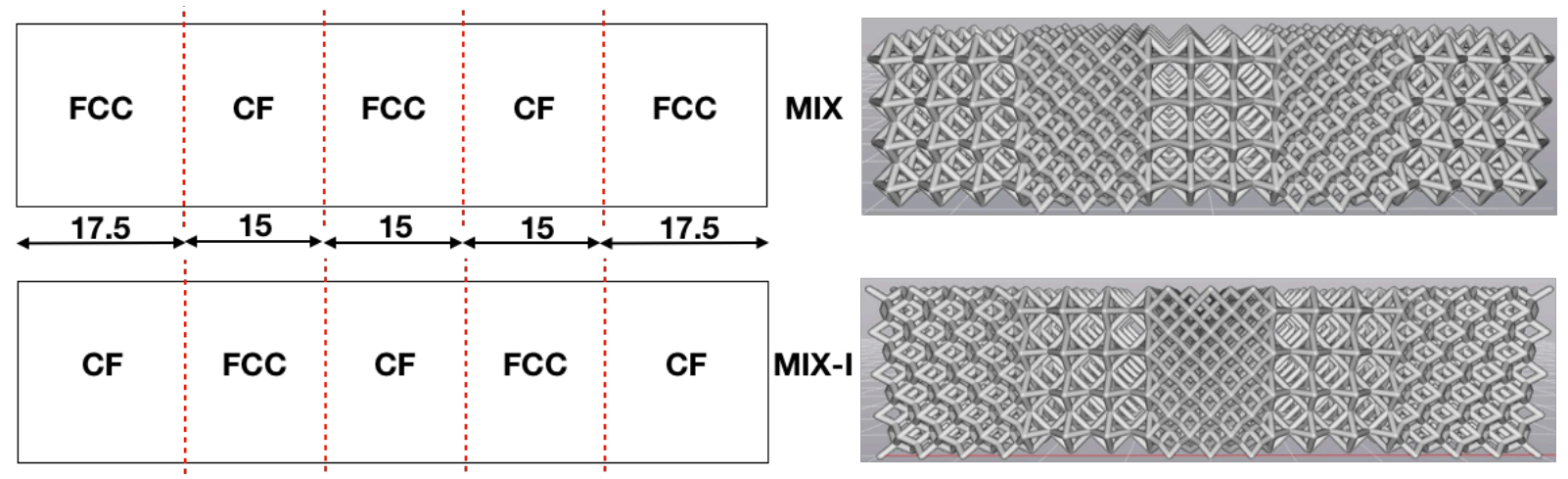

Figure 3. How the design space was split into 5 regions to create $\mathrm{H}-\mathrm{C}$ and $\mathrm{H}-\mathrm{C}+\mathrm{D}$ lattice structures. The MIX-I sample has the same type of unit cells of the MIX (i.e., FCC and CF) but they are placed inverted.

\subsection{Lattice structures prototyping and testing}

As previously mentioned, for prototyping the samples we used the Ultimaker 3 printer and the Ultimaker White PLA filament. We also used the Ultimaker PVA for printing the FCC-O and the MIX-O samples, since the bottom nodes of the first layer of unit cells were not at the same height, because of the heterogeneous distribution of the material. We printed 3 replicas for each type of sample. They were all printed separately to guarantee that each replica was built using the same printing path. We set a $100 \%$ infill and a $0.2 \mathrm{~mm}$ layer height. 


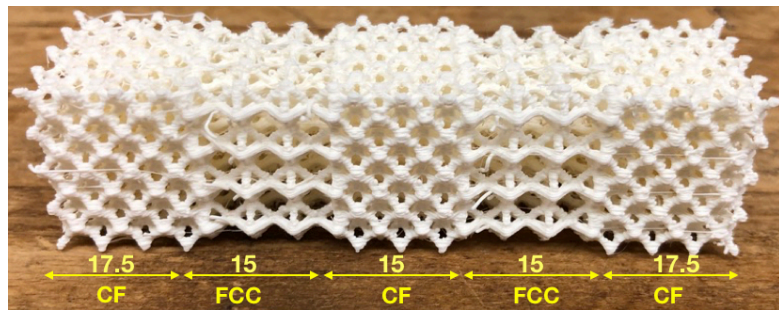

(a)

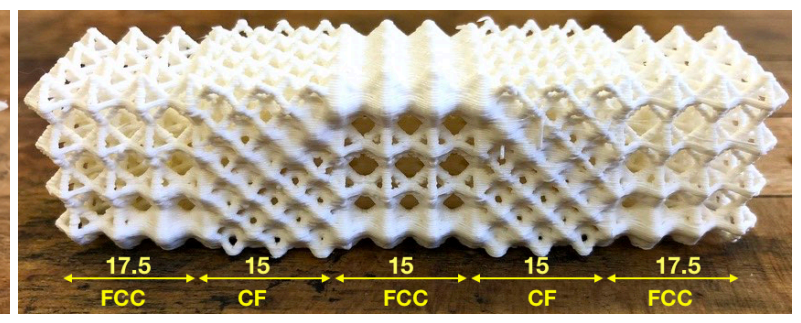

(b)

Figure 4. An example of MIX-I (4a) and MIX-O (4b) samples (see also Table 1 and Figure 3).

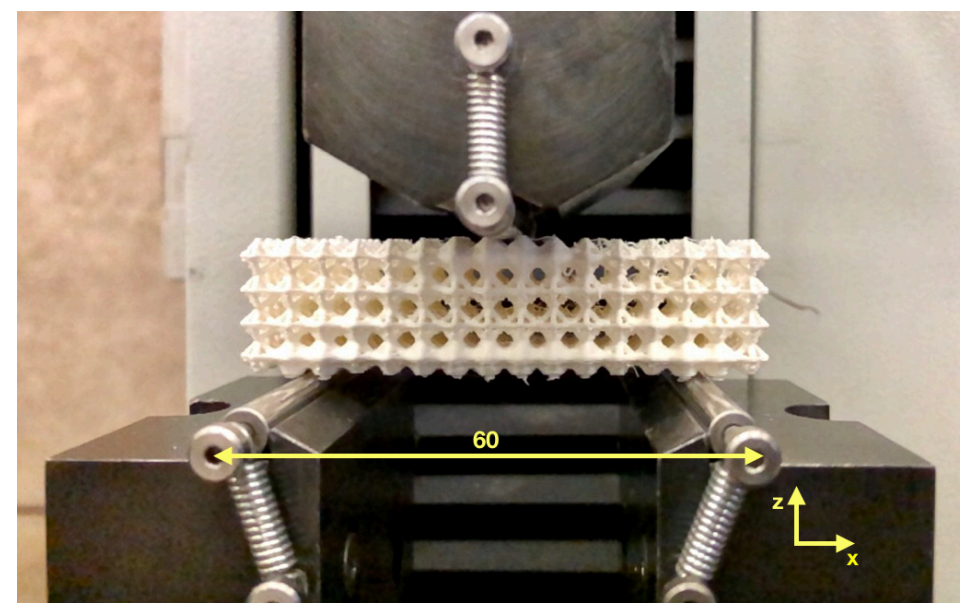

Figure 5. Example of TPB test performed on the FCC-O sample.

For running the TPB test, we used the MTS Synergie 200 (see Figure 5) and the MTS RF 150 machines, setting the span distance at $60 \mathrm{~mm}$, the cross-head speed at $10 \mathrm{~mm} / \mathrm{min}$ and $10 \mathrm{~N}$ of preloading. Cylinders with $6 \mathrm{~mm}$ diameter were used. This set-up replicated the optimisations performed for generating the FCC-O and the MIX-O samples (Table 1).

\section{RESULTS}

Table 2 summarises the main results obtained from the TPB tests while the force vs cross-head displacement curves are represented in Figure 6a. These results are the mean values obtained testing 3 replica for each type of sample. Before discussing these results, it is worth underlying the following aspect. The structures were designed not with the intent of fulfilling a specific functional target but rather to intentionally create different geometric distributions of the material. Specifically, these different distributions were obtained implementing the following strategies: 1) by varying the relative density of the unit cell (FCC_1/2/3, Table 2);2) by varying the relative density within the design space (FCC-O, Table 2); 3) by varying the unit cell geometry (MIX and MIX-I, Table 2); 4) by varying both the unit cell and the relative density within the design space (MIX-O, Table 2).

Results confirmed that it is not only the amount of material used but how the material is distributed, which influences the structure behaviour. If we consider a unit cell and its related boundary conditions, the behaviour of this cell is also determined by its geometry and the spatial variation of its relative density. For example, as amply demonstrated in the literature, the tuning of the material distribution using a variable gradient of density can lead to a more efficient structure: the FCC-O Ultimate Bending Force (UBF) is higher than the FCC_3 UBF even if the MF value of the FCC_3 is higher than the MF value of the FCC-O (Table 2). However, when creating an heterogeneous structure by mixing different type of unit cells, results also demonstrated that we could theoretically obtain the same type of advantage: the MIX and the MIX-I structures have the same beam diameter and the same type of unit cells, but even if the MIX has an MF value lower than the MIX-I, it reached a higher UBF value (Table 2). Using these two results we cannot claim which is the best strategy yet, but we can claim that they both can provide a similar benefit. This means that the proper design of an $\mathrm{H}-\mathrm{C}$ structure can provide a similar effect as an H-D structure. Indeed, the results presented in Table 3 show that, as a consequence 
of the optimisation, the MF value of the MIX-O increased by $46 \%$ compared to the MF value of the MIX, and the UBF value of the MIX-O increased by $347 \%$ compared to the UBF value of the MIX. The MF value of the FCC-O increased by 59\% compared to the FCC_1 (it has the same beam dimension of the MIX), and in this case, the UBF increased by $211 \%$. Hence, the increase of the UBF value is higher for the MIX-O than the FCC-O, while the increase of the MF value is lower. The optimisation process of the two structures is the same, but it is performed starting from two different types of wireframes: HO for the FCC and H-C for the MIX. We can still not claim that the behaviour of the MIX-O is better than FCC-O but, these data demonstrated that the MIX-O is theoretically more efficient than the FCC-O sample. Hence the MIX-O has a more efficient distribution of the material. Finally, this consideration is also supported by Figure 6b: the UBF value of each structure was divided per the related MF value (see also Table 2).

All these results allowed us to derive this additional consideration. An effective strategy to optimise the material distribution inside the design space could be implemented through the following two steps: first by creating the desired heterogeneous wireframe and second by finding the optimal relative density distribution. Nevertheless, further studies will be necessary to understand how to design the geometry of the wireframe properly, and how this wireframe influences the distribution of the relative density.

Table 2. Results of the experimental tests.

\begin{tabular}{lccc}
\hline Sample & Beam $\varnothing(\mathrm{mm})$ & UBF $(\mathrm{N})$ & MF \\
\hline FCC_1 & 1 & 315 & 0.19 \\
FCC_2 & 1.1 & 485 & 0.24 \\
FCC_3 & 1.3 & 970 & 0.32 \\
CF & 1 & 528 & 0.31 \\
MIX & 1 & 440 & 0.25 \\
MIX-I & 1 & 397 & 0.27 \\
MIX-O & $0.8-2.2$ & 1965 & 0.37 \\
FCC-O & $0.8-2.2$ & 981 & 0.30 \\
\hline
\end{tabular}

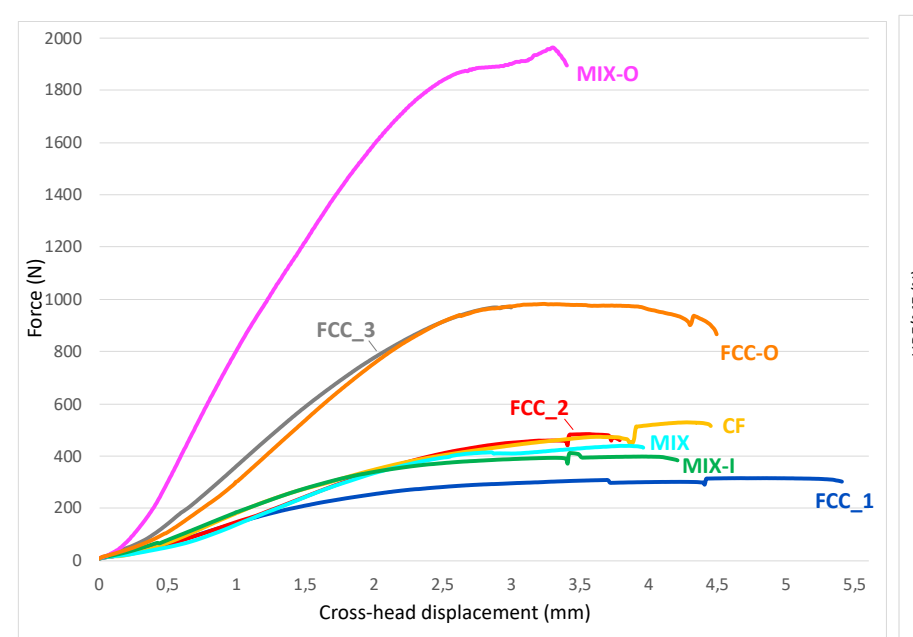

(a)

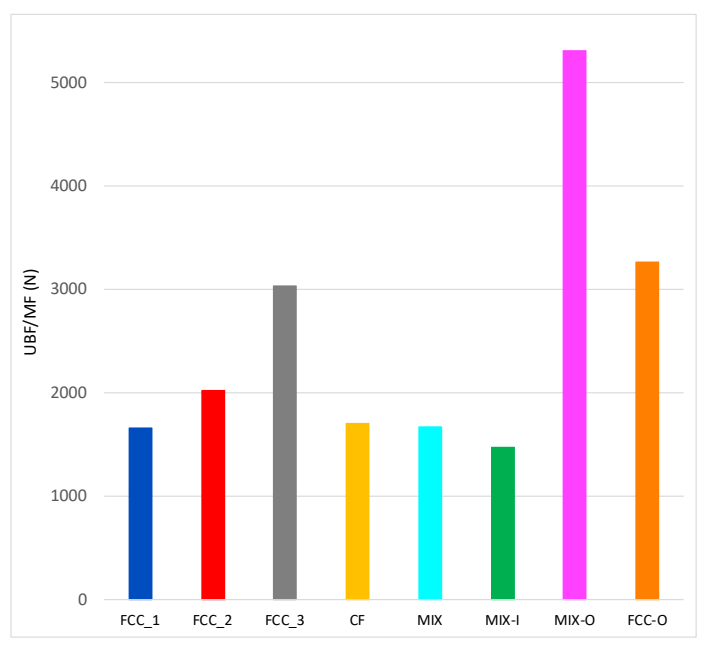

(b)

Figure 6. The force vs cross-head displacement curves obtained from the TPB tests (Figure 6a) and generated using mean values (3 replica were tested for each type of sample). In Figure $6 b$ the UBF values were divided per the related MF value of the structure (see also Table 2).

\section{CONCLUSIONS}

This paper describes an initial exploration of the behaviour of heterogeneous lattice structures (Tang and Zhao, 2018) and of the advantages they provide in further extending design possibilities. We tested three 
Table 3. Comparison between $\mathrm{HO}$ and $\mathrm{H}-\mathrm{D}$ structures and between $\mathrm{H}-\mathrm{C}$ and $\mathrm{H}-\mathrm{C}+\mathrm{D}$ structures.

\begin{tabular}{lcc}
\hline & UBF increase (\%) & MF increase (\%) \\
\hline MIX-O (H-C+D) vs MIX (H-C) & 347 & 46 \\
FCC-O (H-D) vs FCC_1 (HO) & 211 & 59 \\
\hline
\end{tabular}

different types of heterogeneous structures: heterogeneous-density (H-D) structures, created using a unique type of unit cell and a variable density distribution; heterogeneous-cells $(\mathrm{H}-\mathrm{C})$ structures, created by combining different types of unit cell; heterogeneous-cells +density $(\mathrm{H}-\mathrm{C}+\mathrm{D})$ structures, created with different cell geometries and a variable density distribution. Besides, we also tested homogeneous (HO) structures, created using a unique type of unit-cell which is periodically distributed inside the design space.

In this preliminary study we used the following two types of unit cells: Face Centered Cubic (FCC) and Cubic Fluorite (CF). We performed Three-Point Bending (TPB) tests on 3D printed PLA samples. For generating the $3 \mathrm{D}$ models of the lattice structures, we first fixed the design space and the voxel dimensions. Voxels were used to discretise the design space. The H-C and the H-C+D lattice structures were built using the same type of unit cells (i.e., FCC and CF). These cells were not placed randomly: the design space was split into five regions filled, alternatively, with the two unit cells, and taking into account the stress distribution. These unit cells were also used to create HO lattice structures.

The TPB tests confirmed that the material distribution plays a fundamental role in determining the behaviour of the structure. Indeed, as already discussed in the literature, H-D lattice structures can be more efficient than $\mathrm{HO}$ structures having the same unit cell, even with a lower mass fraction value. However, the study also demonstrated that $\mathrm{H}-\mathrm{C}+\mathrm{D}$ structures could lead to more interesting results than $\mathrm{H}-\mathrm{D}$ structures since the heterogeneity of the discretisation of the design space increases the number of possibilities available for distributing the material.

This paper is a preliminary exploration of the potentialities of $\mathrm{H}-\mathrm{C}$ and $\mathrm{H}-\mathrm{C}+\mathrm{D}$ structures. Further studies will be necessary, for example, to understand how to, appropriately, split the design space into functional volumes, assign different types of unit cells, measure and compare the performance of these structures.

\section{REFERENCES}

Ashby, M. (2006), "The properties of foams and lattices", Philosophical Transactions of the Royal Society A: Mathematical, Physical and Engineering Sciences, Vol. 364 No. 1838, pp. 15-30. http://dx.doi.org/10.1098/rsta.2005.1678

Chougrani, L., Pernot, J.-P., Véron, P. and Abed, S. (2018), "Parts internal structure definition using non-uniform patterned lattice optimization for mass reduction in additive manufacturing", Engineering with Computers, Vol. 35 No. 1, pp. 277-289. https://doi.org/10.1007/s00366-018-0598-2

Deng, J. and Chen, W. (2017), "Concurrent topology optimization of multiscale structures with multiple porous materials under random field loading uncertainty", Structural and Multidisciplinary Optimization, Vol. 56 No. 1, pp. 1-19. http://dx.doi.org/10.1007/s00158-017-1689-1

Du, Z., Zhou, X.-Y., Picelli, R. and Kim, H. A. (2018), “Connecting microstructures for multiscale topology optimization with connectivity index constraints”, Journal of Mechanical Design, Vol. 140 No. 11, p. 111417. http://dx.doi.org/10.1115/1.4041176

Hague, R., Campbell, I. and Dickens, P. (2003), "Implications on design of rapid manufacturing”, Proceedings of the Institution of Mechanical Engineers, Part C: Journal of Mechanical Engineering Science, Vol. 217 No. 1, pp. 25-30. https://doi.org/10.1243/095440603762554587

Kolken, H. M. A., Janbaz, S., Leeflang, S. M. A., Lietaert, K., Weinans, H. H. and Zadpoor, A. A. (2018), "Rationally designed meta-implants: a combination of auxetic and conventional meta-biomaterials", Mater. Horiz., Vol. 5, pp. 28-35. http://dx.doi.org/10.1039/C7MH00699C

Li, D., Dai, N., Tang, Y., Dong, G. and Zhao, Y. F. (2019), "Design and optimization of graded cellular structures with triply periodic level surface-based topological shapes”, Journal of Mechanical Design, Vol. 141 No. 7, p. 071402. http://dx.doi.org/10.1115/1.4042617

Liu, L., Yan, J. and Cheng, G. (2008), "Optimum structure with homogeneous optimum truss-like material”, Computers \& Structures, Vol. 86 No. 13-14, pp. 1417-1425. https://doi.org/10.1016/j.compstruc.2007.04.030 
Panesar, A., Abdi, M., Hickman, D. and Ashcroft, I. (2018), "Strategies for functionally graded lattice structures derived using topology optimisation for additive manufacturing", Additive Manufacturing, Vol. 19, pp. 8194. https://doi.org/10.1016/j.addma.2017.11.008

Primo, T., Calabrese, M., Del Prete, A. and Anglani, A. (2017), “Additive manufacturing integration with topology optimization methodology for innovative product design”, The International Journal of Advanced Manufacturing Technology, Vol. 93 No. 1, pp. 467-479. https://doi.org/10.1007/s00170-017-0112-9

Rosen, D. W., Seepersad, C. C., Simpson, T. W. and Williams, C. B. (2015), "Special issue: Design for additive manufacturing: A paradigm shift in design, fabrication, and qualification", Journal of Mechanical Design, Vol. 137 No. 11, pp. 110301-110301-2. http://dx.doi.org/10.1115/1.4031470

Stankovic, T., Mueller, J., Egan, P. and Shea, K. (2015), “A generalized optimality criteria method for optimization of additively manufactured multimaterial lattice structures”, Journal of Mechanical Design, Vol. 137 No. 11, p. 111405. http://dx.doi.org/10.1115/1.4030995

Tamburrino, F., Graziosi, S. and Bordegoni, M. (2018), "The design process of additively manufactured mesoscale lattice structures: A review", Journal of Computing and Information Science in Engineering, Vol. 18 No. 4, p. 040801. http://dx.doi.org/10.1115/1.4040131

Tang, Y. and Zhao, Y. (2015), "Lattice-skin structures design with orientation optimization", 26th Solid Freeform Fabrication Symposium (SFF), Austin, Texas 10-12 August 2015, pp. 1378-1393.

Tang, Y. and Zhao, Y. F. (2018), "Multifunctional design of heterogeneous cellular structures", Structural and Multidisciplinary Optimization, Vol. 58 No. 3, pp. 1121-1138. http://dx.doi.org/10.1007/s00158-018-1956-9

Thompson, M. K., Moroni, G., Vaneker, T., Fadel, G., Campbell, R. I., Gibson, I., Bernard, A., Schulz, J., Graf, P., Ahuja, B. and Martina, F. (2016), "Design for additive manufacturing: Trends, opportunities, considerations, and constraints”, CIRP Annals, Vol. 65 No. 2, pp. 737-760. https://doi.org/10.1016/j.cirp.2016.05.004

Ultimaker Cura (2019). Available at: https://ultimaker.com/en/products/ultimaker-cura-software (accessed 22 March 2019).

Ultimaker PLA technical data sheet (2018). Available at: https://ultimaker.com/en/resources/49911-pla (accessed 22 March 2019).

Wang, C., Zhu, J. H., Zhang, W. H., Li, S. Y. and Kong, J. (2018), “Concurrent topology optimization design of structures and non-uniform parameterized lattice microstructures', Structural and Multidisciplinary Optimization, Vol. 58 No. 1, pp. 35-50. http://dx.doi.org/10.1007/s00158-018-2009-0

Wang, Y., Zhang, L., Daynes, S., Zhang, H., Feih, S. and Wang, M. Y. (2018), "Design of graded lattice structure with optimized mesostructures for additive manufacturing”, Materials \& Design, Vol. 142, pp. 114-123. https://doi.org/10.1016/j.matdes.2018.01.011

Watts, D. M. and Hague, R. J. (2006), "Exploiting the design freedom of RM", 17th Solid Freeform Fabrication Symposium (SFF 2006), Austin, Texas 14-16 August 2006, pp. 656-667.

Xiao, Z., Yang, Y., Xiao, R., Bai, Y., Song, C. and Wang, D. (2018), "Evaluation of topology-optimized lattice structures manufactured via selective laser melting”, Materials \& Design, Vol. 143, pp. 27-37. https://doi.org/10.1016/j.matdes.2018.01.023

\section{ACKNOWLEDGMENTS}

The authors wish to thank the nTopology company (www.ntopology.com) for providing the nTopology Element Pro software. 